\title{
MEMBERS OF THE SOCIETY
}

\author{
January 1, 1920
}

[Life members are designated by *]

Adams, Prof. E. P. Princeton University, Princeton, N. J.

Adams, O. S. Computer, U. S. Coast and Geodetic Survey, Washington, D. C. 325 Seventh Street, N. E.

Agard, Asst. Prof. H. L. Williams College, Williamstown, Mass.

Akers, Prof. O. P. Allegheny College, Meadville, Pa.

AкImoFf, N. W. Engineer. Harrison Building, Philadelphia, Pa.

Alexander, Dr. J. W. 601 West 110th Street, New York, N. Y.

ALEY, Pres. R. J. University of Maine, Orono, Me.

ALGER, P. L. General Electric Company, Schenectady, N. Y. 44 Eleanor Street.

Allardice, Prof. R. E. Stanford University, Calif.

Allen, Asst. Prof. E. S. University of West Virginia, Morgantown, W. Va.

Allen, Dr. FloRENCE E. Instructor, University of Wisconsin, Madison, Wis. 219 Lathrop Street.

Allen, Asso. Prof. Joseph. College of the City of New York, New York, N. Y.

Allen, Prof. R. B. Kenyon College, Gambier, Ohio.

Ames, Asso. Prof. L. D. University of Missouri, Columbia, Mo. 208 Thilly Avenue.

Ammerman, Charles. MeKinley Manual Training High School, St. Louis, Mo.

ANDeregG, Prof. Frederick. Oberlin College, Oberlin, Ohio. 20\% East College Street.

*Archibald, Asso. Prof. R. C. Brown University, Providence, R. I. 9 Charles Field Street.

ARMs, Dr. R. A. Instructor, University of Pennsylvania, Philadelphia, Pa. 25 Graduate House.

Armstrong, Prof. G. N. Ohio Wesleyan University, Delaware, Ohio.

Armstrong, Asst. Prof. L. E. Stevens Institute of Technology, Hoboken, N. J.

AshCramt, Prof. T. B. Colby College, Waterville, Me. 34 Pleasant Street.

Ashton, Prof. C. H. Kansas University, Lawrence, Kan, 1200 Ohio Street. 
*Atchison, Prof. C. S. Washington and Jefferson College, Washington, Pa. 403 East Chestnut Street.

BabB, Asst. Prof. M. J. University of Pennsylvania, Philadelphia, Pa.

*Bacon, Prof. Clara L. Goucher College, Baltimore, Md. 2216 North Calvert Street.

BaILey, Prof. F. H. Massachusetts Institute of Technology, Cambridge, Mass.

BAKer, Asst. Prof. R. P. State University of Iowa, Iowa City, Iowa. 929 Kirkwood Avenue.

BANERJ, Prof. S. K. University of Calcutta, Calcutta, India. 92 Upper Circular Road.

Bareis, Asst. Prof. Grace M. Ohio State University, Columbus, Ohio. 201 West 11th Avenue.

BARNETT, Dr. I. A. Instructor, Harvard University, Cambridge, Mass. 51 Grays Hall.

Barnex, Prof. IDA. Meredith College, Raleigh, N. C.

Barnum, Dr. Charlotte C. 523 West 121st Street, New York, N. Y.

Barrow, Dr. D. F. University Campus, Athens, Ga.

Bartlett, Prof. D. P. Massachusetts Institute of Technology, Cambridge, Mass.

BarTon, Asst. Prof. R. M. University of Minnesota, Minneapolis, Minn.

Barton, Prof. S. M. University of the South, Sewanee, Tenn.

Bateman, Prof. Harry. Throop College, Pasadena, Calif.

Bates, Asso. Prof. W. H. Purdue University, Lafayette, Ind. 403 Russell Street, West Lafayette.

Beal, Asst. Prof. F. W. University of Pennsylvania, Philadelphia, Pa. College Hall.

Beattry, Asst. Prof. SAmues. University of Toronto, Toronto, Canada.

BECKETT, C. H. Actuary, State Life Insurance Company, Indianapolis, Ind.

Beetle, Asst. Prof. R. D. Dartmouth College, Hanover, N. H. $3 \mathrm{Elm}$ Street.

BeLcher, D. R. American Telephone and Telegraph Company, 195 Broadway, New York, N. Y. 272 West 84th Street.

BELL, Asst. Prof. E. T. University of Washington, Seattle, Wash.

Beman, Prof. W. W. University of Michigan, Ann Arbor, Mich. 813 East Kingsley Street.

Benedict, Prof. H. Y. University of Texas, Austin, Tex.

Benedict, Asso. Prof. Suzan R. Smith College, Northampton, Mass. 11 Barrett Place.

*Bennetr, Asso. Prof. A. A. University of Texas, Austin, Tex. ${ }^{1738}$ F Street, N. W., Washington, D. C.

Berg, Prof. E. J. Union College, Schenectady, N. Y.

Bernstein, Asst. Prof. B. A. University of California, Berkeley, Calif. Faculty Club. 
*Berry, Arthur. Fellow and Assistant Tutor, King's College, Cambridge, England. Meadowside, Grantchester Meadows, Cambridge.

Berry, Asso. Prof. W. J. Brooklyn Polytechnic Institute, Brooklyn, N. Y. 224 St. John's Place.

Betz, Herman. Instructor, Sheffield Scientific School, Yale University, New Haven, Conn.

Betz, Willitam. Vice-Principal, East High School, Rochester, N. Y. 160 Grand Avenue.

BigbeE, J. A. High School, Little Rock, Ark. 1611 Rock Street.

BikLt, C. E. Central High School, Syracuse, N. Y.

BiLl, Asst. Prof. E. G. Dartmouth College, Hanover, N. H.

BIRKHoFf, Prof. G. D. Harvard University, Cambridge, Mass. 44 Shepard Street.

BlichFeldT, Prof. H. F. Stanford University, Calif.

${ }^{*}$ BuIss, Prof. G. A. University of Chicago, Chicago, Ill. 5625 Kenwood Avenue.

Blumberg, Asst. Prof. Henry. University of Illinois, Urbana, Il.

BORger, Prof. R. L. Ohio University, Athens, Ohio. 70 University Terrace.

Bose, A. C. Controller of Examinations, University of Calcutta, Calcutta, India. 8 Dinabandhu Lane, Simla Post Office, Calcutta.

Boulad, FarID. Engineer, Bridges Department, State Railways, Cairo, Egypt.

*Bouton, Asso. Prof. C. L. Harvard University, Cambridge, Mass. 9 Avon Street.

Boutroux, Prof. Pierre. Princeton University, Princeton, N. J. Nassau Club.

Bowden, Prof. Joseph. Adelphi College, Brooklyn, N. Y. 24 Clifton Place.

Boxd, Prof. P. P. University of Kentucky, Lexington, Ky.

Brackett, Prof. F. P. Pomona College, Claremont, Calif.

Bradshaw, Asso. Prof. J. W. University of Michigan, Ann Arbor, Mich. 1212 Roosevelt Avenue.

Brainard, Millar. Assistant Vice-President, Central Trust Company of Illinois, Chicago, Ill. 125 West Monroe Street.

Bramble, Asst. Prof. C. C. U. S. Naval Academy, Annapolis, Md. 316 West Street.

Brand, Asso. Prof. Louis. University of Cincinnati, Cincinnati, Ohio. 266 Dorchester Avenue.

Bratton, Prof. W. A. Whitman College, Walla Walla, Wash. $5^{70}$ Boyer Avenue.

BrAY, Dr. H. E. Instructor, Rice Institute, Houston, Tex.

BreCKEnRIDGE, W. E. Associate, Teachers College, Columbia University, New York, N. Y. 21 Sycamore Avenue, Mount Vernon, N. Y.

Brenke, Prof. W. C. University of Nebraska, Lincoln, Neb. 1250 South 21st Street. 
Brink, Asst. Prof. R. W. University of Minnesota, Minneapolis, Minn. 4 Merchiston Avenue, Edinburgh, Scotland.

BRomwich, Dr. T. J. I'A. Lecturer, Cambridge University, Cambridge, England. 1 Selwyn Gardens.

Brooke, Prof. W. E. University of Minnesota, Minneapolis, Minn.

*Brown, Prof. E. W. Yale University, New Haven, Conn. 116 Everit Street.

*Brown, J. S. Consulting Engineer. Far View, P. O. Box 38, Black Hall, Conn.

Brown, Dr. T. H. Instructor, Brown University, Providence, R. I. 79 Taber Avenue.

Buchanan, Prof. Daniet. Queen's University, Kingston, Ontario, Canada. 142 Stuart Street.

Buchanan, Prof. H. E. University of Tennessee, Knoxville, Tenn. Army Y. M. C. A., Building 323, Fort Oglethorpe, Ga.

Buck, Asst. Prof. Thomas. University of California, Berkeley, Calif. Faculty Club.

*Bullard, Prof. W. G. Syracuse University, Syracuse, N. Y. 117 Redfield Place.

*Burgess, Dr. H. T. 37 Beers Street, New Haven, Conn.

Burgess, Asst. Prof. R. W. Brown University, Providence, R. I.

Bussey, Asso. Prof. W. H. University of Minnesota, Minneapolis, Minn.

Butts, Jun. Prof. W. H. University of Michigan, Ann Arbor, Mich. 9.19 Oakland Avenue.

Byerly, Prof. W. E. Harvard University, Cambridge, Mass. 39 Hammond street.

CaIn, Prof. William. University of North Carolina, Chapel Hill, N. C Carrns, Asso. Prof. W. D. Oberlin College, Oberlin, Ohio. 27 King Street.

Cajori, Prof. Florian. University of California, Berkeley, Calif.

Calmoun, Asso. Prof. J. W. University of Texas, Austin, Tex. 2805 Rio Grande Street.

*CAmp, Prof. B. H. Wesleyan University, Middletown, Conn. 150 High Street.

CAMP, Dr. C. C. Instructor, University of Illinois, Urbana, Ill. 60\% West Nevada Street.

CAMpbell, Prof. D. F. Armour Institute of Technology, Chicago, Ill.

Campbell, Dr. G. A. Research Engineer, American Telephone and Telegraph Company, 195 Broadway, New York, N. Y.

CAMpbell, Dr. J. W. Associate, State University of Iowa, Iowa City, Iowa.

Candy, Prof. A. L. University of Nebraska, Lincoln, Neb. Station A.

Caparo, Prof. J. A. Notre Dame University, Notre Dame, Ind. P. $O$. Box 54 . 
Capron, Prof. Paul. U. S. Naval Academy, Annapolis, Md. 139 Market Street.

CAREY, Asst. Prof. E. F. A. University of Montana, Missoula, Mont. 310 McLeod Avenue.

Carmichaed, Asso. Prof. R. D. University of lllinois, Urbana, Ill.

CARPENter, Asso. Prof. A. F. University of Washington, Seattle, Wash.

CARruth, Prof. W. M. Hamilton College, Clinton, N. Y. P. O. Box 25.

Carson, J. R. Research Engineer, American Telephone and Telegraph Company, 195 Broadway, New York, N. Y.

Carter, Asst. Prof. B. E. Colby College, Waterville, Me.

Carver, Asst. Prof. W. B. Cornell University, Ithaca, N. Y. White Hall.

Chace, Dr. A. B. Chancellor, Brown University, Providence, R. I. 324 Angell Street.

Chadbourne, Miss Martha F. Dana Hall, Wellesley, Mass.

Chambers, Asst. Prof. G. G. University of Pennsylvania, Philadelphia, Pa. 79 Drexel Avenue, Lansdowne, $P a$.

Chittenden, Asst. Prof. E. W. State University of Iowa, Iowa City, Iowa. 221 Physics Building.

Chitrtenden, Prof. J. B. Brooklyn Polytechnic Institute, Brooklyn, N. Y.

Clark, Prof. J. E. Yale University, New Haven, Conn. 193 Longmeadow Street, Springfield, Mass.

Clarke, Prof. E. H. Hiram College, Hiram, Ohio.

Clements, Asst. Prof. G. R. U. S. Naval Academy, Annapolis, Md.

Coвв, Asso. Prof. C. W. Amherst College, Amherst, Mass. 75 South Pleasant Street.

Coвв, Prof. H. E. Lewis Institute, Chicago, Ill.

CoBls, Prof. A. B. University of Illinois, Urbana, Ill.

CoE, Asst. Prof. IC. J. University of Michigan, Ann Arbor, Mich. 1020 Michigan Avenue.

Cofris, Dr. J. G. Director of Aeronautical Research, Curtiss Engineering Corporation, Garden City, L. I.

Cohen, Asso. Prof. ABRAham. Johns Hopkins University, Baltimore, Md.

Cohen, Dr. Teresa. 1709 Linden Avenue, Baltimore, Ma.

*Cole, Prof. F. N. Columbia University, New York, N. Y.

ColpitTs, Asso. Prof. E. C. State College of Washington, Pullman, Wash. 410 Montgomery Street.

Colpitts, Asso. Prof. Julia T. Iowa State College, Ames, Iowa. 219 Ash Avenue.

Comstock, Prof. C. E. Bradley Polytechnic Institute, Peoria, Ill.

CoNwELL, Dr. G. M. Instructor, New York State College for Teachers, Albany, N. Y.

Conweil, Asso. Prof. H. H. University of Idaho, Moscow, Idaho. 618 South Washington Street. 
*Coolidge, Prof. J. L. Harvard University, Cambridge, Mass. 27 Fayerweather Street.

*Copeland, Dr. Lennie P. Instructor, Wellesley College, Wellesley, Mass. 86 Shafer Hall.

Corey, S. A. 1079 23d Street, Des Moines, Iowa.

CourT, Asst. Prof. N. A. University of Oklahoma, Norman, Okla. 707 Jenkins Avenue.

Cowlex, Asso. Prof. Elizabeth B. Vassar College, Poughkeepsie, N. Y.

Cox, Dr. L. C. Statistician, War Department, Washington, D. C. 1234 Crittenden Street, $N$. W.

CRaIG, Asst. Prof. C. F. Cornell University, Ithaca, N. Y. $311 \mathrm{Elm}$ wood Avenue.

Crathorne, Asst. Prof. A. R. University of Illinois, Champaign, Ill. 1113 South Fourth Street.

Crawlex, Prof. E. S. University of Pennsylvania, Philadelphia, Pa.

Cresse, Dr. G. H. Instructor, U. S. Naval Academy, Annapolis, Md. 69 Conduit Street.

Cromwell, J. W., Jr. Dunbar High School, Washington, D. C. 1815 13th Street.

Cronin, Dr. S. Elizabeth. Equitable Life Assurance Company, 120 Broadway, New York, N. Y.

CRUM, Dr. W. L. Instructor, Yale University, New Haven, Conn. 766 Yale Station.

Cummings, Asso. Prof. Louise D. Vassar College, Poughkeepsie, N. Y.

${ }^{*}$ Cunningham, Lieut. Col. A. J. C. Fellow, King's College, London, England. 20 Essex Villas, Kensington.

CurJel, H. W. Actuary, Illinois Life Insurance Company, Otis Building, 10 South La Salle Street, Chicago, Ill.

Currier, Asst. Prof. C. H. Brown University, Providence, R. I.

Curtis, A. M. State Normal School, Oneonta, N. Y.

CURTIS, Dr. H. B. Instructor, Northwestern University, Evanston, Ill. 620 University Place.

Curtis, Dr. Mary F. Instructor, Wellesley College, Wellesley, Mass. 1 Waban Street.

CuRTISs, Prof. D. R. Northwestern University, Evanston, Ill. 2023 Sherman Avenue.

Dalaker, Asst. Prof: H. H. University of Minnesota, Minneapolis, Minn. 523 Walnut Street, S. $E$.

Daniell, Asst. Prof. P. J. Rice Institute, Houston, Tex.

Dantzig, Dr. ToBias. Instructor, Johns Hopkins University, Baltimore, Ma.

*Davis, Prof. H. N. Harvard University, Cambridge, Mass. 19 Ash Street.

Davis, Prof. J. M. University of Kentucky, Lexington, Ky. 340 Madison Place. 
Davis, Prof. N. F. Brown University, Providence, R. I. 19 Ash Street, Cambridge, Mass.

Davisson, Prof. S. C. Indiana University, Bloomington, Ind. 515 East Third Street.

Decherd, Miss M. E. Instructor, University of Texas, Austin, Tex. 2313 Nueces Street.

DeCKer, Prof. F. F. Syracuse University, Syracuse, N. Y. 312 Marshall Street.

De Cov, Prof. E. E. University of Oregon, Eugene, Ore. 1135 Mill Street.

*DederICK, Dr. L. S. Instructor, U. S. Naval Academy, Annapolis, Md. 306 West Street.

Defoe, Prof. L. M. University of Missouri, Columbia, Mo. 810 Virginia Avenue.

Deimel, Asst. Prof. R. F. Stevens Institute of Technology, Hoboken, N. J.

DeLong, Prof. I. M. University of Colorado, Boulder, Colo. 1341 Broadway.

DeLury, Prof. A. T. University of Toronto, Toronto, Canada.

*Dennetr, Dr. W. S. 36 Gramerey Park, New York, N. Y.

Denton, Dr. W. W. Instructor, University of Michigan, Ann Arbor, Mich. 734 East University Avenue.

DePorte, Asst. Prof. J. V. New York State College for Teachers, Albany, N. Y.

Dickson, Prof. L.'E. University of Chicago, Chicago, Ill. 5535 University Avenue.

Dimrck, C. E. Instructor, U. S. Coast Guard Academy, New London, Conn. 47 Squire Street.

Dines, Asst. Prof. C. R. Dartmouth College, Hanover, N. H.

Dines, Prof. L. L. University of Saskatchewan, Saskatoon, Saskatchewan, Canada.

Dodd, Asso. Prof. E. L. University of Texas, Austin, Tex. 3012 West Avenue.

Dowling, Asso. Prof. L. W. University of Wisconsin, Madison, Wis. 2 Roby Road.

Dresden, Asst. Prof. ARNold. University of Wisconsin, Madison, Wis. 2114 Vilas Street.

Dunkel, Asso. Prof. Отто. Washington University, St. Louis, Mo.

Durell, Dr. Fletcher. Lawrenceville School, Lawrenceville, N. J.

Durfee, Prof. W. P. Hobart College, Geneva, N. Y. 639 Main Street.

Duval, Asso. Prof. E. P. R. University of Oklahoma, Norman, Okla. 427 West Boyd Street.

Dysart, Robert. Certified Public Accountant. 40 State Street, Boston, Mass. 
EAgLes, Prof. T. R. Howard College, Birmingham, Ala. 8016 Underwood Avenue.

EARLe, Prof. M. D. Furman University, Greenville, S. C.

Echols, Prof. C. P. U. S. Military Academy, West Point, N. Y.

ECHols, Prof. W. H. University of Virginia, University, Va.

EDDY, Prof. H. T. University of Minnesota, Minneapolis, Minn. 916 Sixth Street, S. $E$.

Edington, W. E. University of Illinois, Urbana, Ill. 1114 West Illinois Street.

EDMondson, Prof. T. W. New York University, University Heights, New York, N. Y.

Edwards, Prof. G. C. University of California, Berkeley, Calif. 2546 Dana Street.

Eells, Prof. W. C. Whitman College, Walla Walla, Wash. 1134 Alvarado Terrace.

EIEsland, Prof. JoHn. University of West Virginia, Morgantown, W. Va.

*Eisenhart, Prof. L. P. Princeton University, Princeton, N. J. 25 Alexander Street.

*Elliotr, Prof. E. B. Oxford University, Oxford, England. 4 Bardwell Road.

Emch, Asso. Prof. ARNold. University of Illinois, Urbana, Ill. 502 West Oregon Street.

Emmons, Prof. C. W. Simpson College, Indianola, Iowa.

Emmons, Asst. Prof. L. C. Michigan Agricultural College, East Lansing, Mich.

ENGLISH, HARRY. Head of Mathematical Department, Washington High Schools, Washington, D. C. 2907 P Street, N. W.

EPPERSON, Asso. Prof. C. A. First District Normal School, Kirksville, Mo.

Epsteen, Dr. Saul. First State Bank, Clifton, Colo.

*Escott, E. B. 822 Oakwood Boulevard, Chicago, IIl.

Estr, Prof. T. C. Amherst College, Amherst, Mass. 40 Dana Street.

Etrlinger, Adj. Prof. H. J. University of Texas, Austin, Tex. 2910 Harris Park Avenue.

*Evans, Prof. G. C. Rice Institute, Houston, Tex.

Evans, G. W. Headmaster, Charlestown High School, Boston, Mass. 31 Broad Street, Lynn, Mass.

Evans, Prof. H. B. University of Pennsylvania, Philadelphia, Pa. College Hall.

FAught, Prof. J. B. State Normal College, Kent, Ohio. 226 Lincoln Avenue.

*FerRY, Pres. F. C. Hamilton Collegé, Clinton, N. Y.

Field, Prof. Floxd. Georgia School of Technology, Atlanta, Ga. Route 1, Decatur, Ga. 
FIELD, Prof. PeTER. University of Michigan, Ann Arbor, Mich. 904 Olivia Avemue.

*Fields, Prof. J. C. University of Toronto, Toronto, Canada.

Findlay, Prof. William. McMaster University, Toronto, Canada.

Fine, Prof. H. B. Princeton University, Princeton, N. J.

Finkel, Prof. B. F. Drury College, Springfield, Mo.

${ }^{*}$ Fischer, Prof. C. A. Trinity College, Hartford, Conn.

Fisher, Prof. G. E. University of Pennsylvania, Philadelphia, Pa.

Fisher, Prof. Irving. Yale University, New Haven, Conn. 460 Prospect Street.

*FISKE, Prof. T. S. Columbia University, New York, N. Y.

Fitch, Mrs. Edward. Clinton, N. Y.

*Fite, Prof. W. B. Columbia University, New York, N. Y.

Fitrerer, Prof. J. C. University of Wyoming, Laramie, Wyo. 511 South 11th Street.

FitzPatrick, T. J. Station A, Lincoln, Neb.

FlaAmmer, Asst. Prof. ERNest. Queen's University, Kingston, Ontario, Canada.

Focke, Prof. T. M. Case School of Applied Science, Cleveland, Ohio.

FoRd, Dr. L. R. Instructor, Harvard University, Cambridge, Mass. 16 Wendell Street.

*Ford, Prof. W. B. University of Michigan, Ann Arbor, Mich. 904 Forest Avenue.

Forsyth, Prof. A. R. Imperial College of Science and Technology, London, S. W., England.

Forster, Asst. Prof. C. H. Dartmouth College, Hanover, N. H. Thayer Lodge.

ForT, Prof. TomLinson. University of Alabama, University, Ala.

FrankLin, Dr. Fabian. 617 West 113th Street, New York, N. Y.

FrizeLL, Dr. A. B. 164 West 65 th Street, New York, N. Y.

FrX, T. C. Research Engineer, Western Electric Company, 463 West Street, New York, N. Y.

GABA, Asso. Prof. M. G. University of Nebraska, Lincoln, Neb.

GALE, Prof. A. S. University of Rochester, Rochester, N. Y.

GarIs, Prof. C. F. F. Union College, Schenectady, N. Y. 226 Parkwood Boulevard.

Garretson, Asst. Prof. W. V. N. Rutgers College, New Brunswick, N. J. 586 George Street.

Garrett, Prof. W. H. Baker University, Baldwin, Kan.

Garrison, Prof. W. A. King College, Bristol, Tenn.-Va.

GAVETT, Asst. Prof. G. I. University of Washington, Seattle, Wash. 1402 East 75th Street.

*GerRans, H. T. Fellow and Lecturer, Worcester College, Oxford, England. 20 St. John Street. 
Gibson, Prof. G. A. University of Glasgow, Glasgow, Scotland, 10 The University.

GIBson, Prof. J. L. University of Utah, Salt Lake City, Utah.

GILlesPIE, Asst. Prof. D. C. Cornell University, Ithaca, N. Y. Cayuga Heights.

Glasgow, Dr. Josephine B. Instructor, University of Illinois, Urbana, Ill. 924 West Illinois Street.

Glashan, Dr. J. S. C. 68 Somerset Street, West, Ottawa, Canada.

GLAZIER, Prof. Harriet E. Western College for Women, Oxford, Ohio.

Glenn, Prof. O. E. University of Pennsylvania, Philadelphia, Pa. 127 McKinley Avenue, Lansdowne, $P a$.

GLover, Prof. J. W. University of Michigan, Ann Arbor, Mich. 620 Oxford Road.

Gossard, Asst. Prof. H. C. University of Oklahoma, Norman, Okla.

Gottschali, Louis. 251 West 89th Street, New York, N. Y.

*Gould, Miss Alice B. Care of W. W. Vaughn, Esq., Exchange Building, Boston, Mass.

Graber, Prof. M. E. Morningside College, Sioux City, Iowa. 4005 Morningside Avenue.

GrahaM, W. J. Actuary, Equitable Life Assurance Society, 120 Broadway, New York, N. Y.

Granville, Pres. W. A. Pennsylvania College, Gettysburg, Pa.

Graustein, Asst. Prof. W. C. Harvard University, Cambridge, Mass. 19 Arlington Street.

Gravatt, T. E. Instructor, Pennsylvania State College, State College, Pa.

Graves, Dr. G. H. Instructor, Purdue University, Lafayette, Ind. 234 Pierce Street, West Lafayette.

*Greenhill, Sir George. 1 Staple Inn, London, West, England.

Grennan, Dr. ElizabeTh B. (Mrs. JoHn). Instructor, University of Illinois, Champaign, Ill. 504 Chalmers Street.

GRIFrin, Prof. F. L. Reed College, Portland, Ore.

${ }^{*}$ GroAt, B. F. Consulting Engineer. 2400 Oliver Building, Pittsburgh, $\mathrm{Pa}$.

GRonwall, Dr. T. H. Mathematics and Dynamics Expert, Bureau of Ordnance, War Department, Washington, D. C. B-1-109, 6th and B Streets.

Grove, Dr. C. C. 3129 Broadway, New York, N. Y.

GuMmer, Asst. Prof. C. F. Queen's University, Kingston, Ontario, Canada. 143 Collingwood Street.

${ }^{*}$ Gummere, Prof. H. V. Drexel Institute, Philadelphia, Pa. Glen Mills, Delaware County, Pa.

Gundersen, Prof. Carl. Oklahoma Agricultural and Mechanical College, Stillwater, Okla. 217 College Avenue.

Gunther, Prof. C. O. Stevens Institute of Technology, Hoboken, N. J. P. O. Box $7 \%$. 
Hall, Prof. A. G. University of Michigan, Ann Arbor, Mich. 1036 Oalcland Avenue.

Halperin, Asst. Prof. Hrlled. University of Arkansas, Fayetteville, Ark.

*Halsted, Dr. G. B. 904 Ninth Avenue, Greeley, Colo.

*Hanawalt, Prof. F. W. College of Puget Sound, Tacoma, Wash. 826 North Steele Street.

HaNcock, Prof. Harris. University of Cincinnati, Cincinnati, Ohio. 2365 Auburn Avenue.

Hanna, Asso. Prof. U. S. Indiana University, Bloomington, Ind.

*Hardcastle, Miss Frances. 3 Osborne Terrace, Newcastle-on-Tyne, England.

Harding, Prof. A. M. University of Arkansas, Fayetteville, Ark. 537 Leverett Street.

HaRDY, G. H. Fellow and Lecturer, Trinity College, Cambridge, England.

*Hardy, Prof. J. G. Williams College, Williamstown, Mass.

*Harkness, Prof. James. McGill University, Montreal, Canada.

Harshbarger, Prof. W. A. Washburn College, Topeka, Kan. 1401 College Avenue.

Hart, Asst. Prof. W. L. University of Minnesota, Minneapolis, Minn.

Hart, Asst. Prof. W. W. University of Wisconsin, Madison, Wis.

Haseman, Prof. Charles. University of Nevada, Reno, Nev.

Haseman, Dr. Mary G. Linton, Ind.

HASkell, Prof. M. W. University of California, Berkeley, Calif. P. $O$. Box 3.

Haskins, Prof. C. N. Dartmouth College, Hanover, N. H. Hanover Road, Lebanon, N. H.

Hassler, Dr. J. O. Crane Junior College, Chicago, Inl. 2337 West 108th Place.

Hathaway, Prof. A. S. Rose Polytechnic Institute, Terre Haute, Ind.

Hawkes, Prof. H. E. Columbia University, New York, N. Y.

HAWKESWORTH, Rev. A. S. Mathematician, Bureau of Ordnance, Navy Department, Washington, D. C. 1807 19th Street, N.W.

Hayashi, Prof. Tsuruichi. College of Science, Tôhoku Imperial University, Sendai, Japan.

Hazlett, Asst. Prof. Olive C. Mount Holyoke College, South Hadley, Mass.

Heal, W. E. Computer, Bureau of Plant Industry, Washington, D. C. $3^{77} 4^{7}$ Huntington Street, $N . W$.

HeBBeRt, Dr. C. M. Instructor, University of Illinois, Urbana, Ill. 250 Natural History Hall.

HedRICK, Prof. E. R. University of Missouri, Columbia, Mo.

Henderson, Robert. Actuary, Equitable Life Assurance Society, 165 Broadway, New York, N. Y. 
Hennel, Asst. Prof. Cora B. Indiana University, Bloomington, Ind. 822 East Third Street.

Hewes, Dr. L. I. General Inspector, U. S. Bureau of Public Roads. Post Office Building, Portland, Ore.

Hildembrandt, Asst. Prof. T. H. University of Michigan, Ann Arbor, Mich. 819 South State Street.

Hiltebeitel, Dr. A. M. Trappe, Montgomery County, Pa.

Himwich, Dr. A. A. 1913 Madison Avenue, New York, N. Y.

Hiтchсоск, Prof. R. R. University of North Dakota, University, N. Dak.

Ho, Y. H. Cornell University, Ithaca, N. Y. 204 Fairmount Avemue.

HODGE, F. H. Instructor, Purdue University, Lafayette, Ind. 518 Russell Street, West Lafayette.

Hodgkins, Prof. H. L. George Washington University, Washington, D. C.

Hogrefe, L. T. W. Consulting Chemist. 5403 Illinois Avenue, Washington, D. C.

Holder, Prof. F. J. Mercer University, Macon, Ga. 1302 Elm Street.

Holgate, Prof. T. F. Northwestern University, Evanston, Ill. 617 Library Street.

HollCropr, Prof. T. R. Wells College, Aurora, N. Y.

Hopkins, Asst. Prof. L. A. University of Michigan, Ann Arbor, Mich.

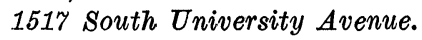

Horton, Dr. Goldie P. Instructor, University of Texas, Austin, Tex. 504 West 32d Street.

Hoskins, Prof. L. M. Stanford University, Calif. 365 Lincoln Avenue, Palo Alto, Calif.

Howe, Prof. H. A. University of Denver, Denver, Colo. 2201 South Fillmore Street.

Howland, Prof. L. A. Wesleyan University, Middletown, C'onn. 34 Home Avenue.

Hoyт, R. S. Research Engineer, American Telephone and Telegraph Company, 195 Broadway, New York, N. Y.

Hulburs, Prof. L. S. Johns Hopkins University, Baltimore, Md.

Huntington, Prof. E. V. Harvard University, Cambridge, Mass. 27 Everett Street.

HuRwitz, Asst. Prof. W. A. Cornell University, Ithaca, N. Y. 8 White Hall.

Hussey, Prof. W. J. University of Michigan, Ann Arbor, Mich.

Hutchinson, Prof. J. I. Cornell University, Ithaca, N. Y. 140 Thurston Avenue.

INCE, Dr. E. L. 6 Moredun Square, Perth, Scotland.

IngoLd, Asst. Prof. Lours. University of Missouri, Columbia, Mo. 206 Thilly Avenue. 
Irwin, Asst. Prof. Frank. University of California, Berkeley, Calif. 1625 Arch Street.

*Jackson, Prof. Dunham. University of Minnesota, Minneapolis, Minn. JACKSON, Dr. L. L. Principal, Baldwin Street School, Montclair, N. J. 17 Oxford Street.

JAcoBUs, D. S. Advisory Engineer, Babcock and Wilcox Company, 85 Liberty Street, New York, N. Y.

James, Asst. Prof. Glenn. Carnegie Institute of Technology, Pittsburgh, Pa. 1027 Murray Hill Avenue.

JAMEs, Asso. Prof. G. O. Washington University, St. Louis, Mo.

Jorre, S. A. Assistant Actuary, Mutual Life Insurance Company, 32 Nassau Street, New York, N. Y.

Johnson, Prof. R. A. Hamline University, St. Paul, Minn.

Johnson, Prof. W. W. U. S. Naval Academy, Annapolis, Md. 909 St. Paul Street, Baltimore, Md.

JoNes, Dr. C. C. Chancellor, University of New Brunswick, Fredericton, N. B., Canada.

Jones, Prof. E. H. Southern Methodist University, Dallas, Tex.

Jones, Asst. Prof. J. L. Syracuse University, Syracuse, N. Y. 842 Ackerman Avenue.

JoRdAN, Asst. Prof. H. E. Kansas University, Lawrence, Kan. 1600 Kentucky street.

KARPINSKI, Prof. L. C. University of Michigan, Ann Arbor, Mich. 1315 Cambridge Road.

*Kasner, Prof. Edward. Columbia University, New York, N. Y. 22 West 119th Street.

KEAN, H. P. 353 West 121st Street, New York, N. Y.

KEFFER, RALPH. Actuarial Department, Group Division, Aetna Life Insurance Company, Hartford, C'onn.

KellogG, Prof. O. D. University of Missouri, Columbia, Mo. 48 Garden Street, Cambridge, Mass.

Kells, Dr. L. M. Instructor, U. S. Naval Academy, Annapolis, Md. 312 West Street.

Kempner, Asst. Prof. A. J. University of Illinois, Urbana, Ill.

Kennelly, Prof. A. E. Harvard University and Massachusetts Institute of Technology, Cambridge, Mass.

*Kenyon, Prof. A. M. Purdue University, Lafayette, Ind. 315 University Street, West Lafayette.

*Keysfr, Prof. C. J. Columbia University, New York, N. Y.

KrLlaAm, Asst. Prof. S. D. University of Alberta, Edmonton South, Alberta, Canada.

Kindle, Asst. Prof. J. H. University of Cincinnati, Cincinnati, Ohio.

Kingston, Asst. Prof. H. R. University of Manitoba, Winnipeg, Manitoba, Canada. 
KIRCheR, Dr. E. A. T. National City Company, 55 Wall Street, New York, N. Y. 1007 West Eighth Street, Plainfield, N. J.

KLINe, Dr. J. R. Associate, University of Illinois, Urbana, Ill. 606 South Goodwin Street.

Knebelman, Asst. Prof. M. S. Lehigh University, South Bethlehem, Pa. 415 Cherokee Street.

Kniselt, Alexander. Columbia City, Ind.

Krathwohl, Asso. Prof. W. C. Armour Institute of Technology, Chicago, Ill.

Kuнn, Prof. H. W. Ohio State University, Columbus, Ohio. 1145 Fairview Avenue, Route 133.

*Kuschke, Dr. C. G. P. 1293 O'Farrell Street, San Francisco, Calif.

KÜSTERMaNn, Dr. W. W. Associate, University of Illinois, Urbana, Ill. 707 South Race Street.

*Ladue, Pomeroy. 185 Canfield Avenue, West, Detroit, Mich.

LAMBerT, Prof. P. A. Lehigh University, South Bethlehem, Pa. 323 North Center Street, Bethlehem.

Lambert, W. D. U. S. Coast and Geodetic Survey, Washington, D. C.

Lamond, Prof. J. K. Pennsylvania College, Gettysburg, Pa. 6023 Catherine Street, Philadelphia, $P$ a.

Lamson, Dr. K. W. Instructor, Columbia University, New York, N. Y.

Landis, Prof. W. W. Dickinson College, Carlisle, Pa.

LANDrY, Prof. A. E. Catholic University of America, Washington, D. C. 3624 13th Street, Brookland, D. C.

Lane, Asst. Prof. E. P. University of Wisconsin, Madison, Wis.

Langman, Harry. Instructor, College of the City of New York, New York, N. Y. 35 West 61st Street.

LAREW, Prof. GILLIE A. Randolph-Macon Woman's C'ollege, Lynchburg, Va.

Laslex, Asst. Prof. J. W., Jr. University of North Carolina, Chapel Hill, N. C. 200 Union Avenue, Burlington, N. C.

Lates, Asso. Prof. Kurt. University of Chicago, Chicago, Ill. 5611 Kenwood Avenue.

Leavens, D. H. Instructor, College of Yale in China, Changsha, China.

Lefschetz, Asst. Prof. Solomon. Kansas University, Lawrence, Kan. 937 Missouri Street.

LEHMER, Prof. D. N. University of California, Berkeley, Calif. 2736 Regent Street.

Leib, Prof. D. D. Connecticut College for Women, New London, Conn. 358 Mohegan Avenue.

Lennes, Prof. N. J. University of Montana, Missoula, Mont.

LeONARD, Prof. H. B. University of Arizona, Tucson, Ariz.

Lester, Prof. O. C. University of Colorado, Boulder, Colo. 1061 11th Street. 
LeStouranon, Asst. Prof. Flora E. Carleton C'ollege, Northfield, Minn. 201 Winona Street.

Leuschner, Prof. A. O. University of California, Berkeley, Calif. National Research Council, 1023 16th Street, N.W., Washington, D. C.

Levi-Civita, Prof. Tullio. University of Rome, Rome, Italy. Piazza S. Bernardo, 106, Rome, 5.

Lewis, Asso. Prof. Florence P. Goucher College, Baltimore, Md. 2435 North Charles Street.

LibBy, B. B. High School and Junior College, San Diego, Calif.

Light, Asso. Prof. G. H. University of Colorado, Boulder, Colo. 958 Pleasant Street.

Linehan, Asst. Prof. P. H. College of the City of New York, New York, N. Y. 346 Convent Avenue.

LING, Prof. G. H. University of Saskatchewan, Saskatoon, Saskatchewan, Canada.

Linton, M. A. Associate Actuary, Provident Life and Trust Company, 409 Chestnut Street, Philadelphia, Pa.

LIPKA, Asst. Prof. JoSEPH. Massachusetts Institute of Technology, Cambridge, Mass.

Locke, L. L. 950 St. John's Place, Brooklyn, N. Y.

Longley, Asst. Prof. W. R. Sheffeld Scientific School, Yale University, New Haven, Conn. 595 Orange Street.

Love, Prof. A. E. H. Oxford University, Oxford, England. 34 St. Margaret's Road.

Love, Asst. Prof. C. E. University of Michigan, Ann Arbor, Mich. 1527 South University Avenue.

*Lovett, Pres. E. O. Rice Institute, Houston, Tex.

LuBy, Prof. W. A. Kansas City Junior College, Kansas City, Mo. 322 South Lawn Street.

Luck, Asso. Prof. J. J. University of Virginia, University, Va. Colonnade $C l u b$.

*LudLOW, Col. H. H., U. S. A. Fort Stevens, Ore.

LunN, Asso. Prof. A. C. University of Chicago, Chicago, Ill. 5211 Kenwood Avenue.

Lyman, Prof. E. A. Michigan State Normal School, Ypsilanti, Mich. 126 North Washington Street.

Lytle, Dr. E. B. Associate, University of Illinois, Urbana, Ill. 903 South Busey Avenue.

McCain, Prof. Gertrude I. Oxford College, Oxford, Ohio.

McClenon, Prof. R. B. Grinnell College, Grinnell, Iowa. 1512 Fourth Avenue.

MCDonnell, John. Computer, Geodetic Survey of Canada. 84 Marlborough Avenue, Ottawa, Canada.

McEwen, Dr. G. F. Oceanographer, Scripps Institution of the University of California, La Jolla, Calif. P. O. Box 68 . 
McKelvey, Asst. Prof. J. V. Iowa State C'ollege, Ames, Iowa. Lock Box 232, Station A.

MCKinnex, Prof. T. E. University of South Dakota, Vermillion, S. Dak. 222 North University Street.

McMackin, Dr. F. J. 11 Lexington Avenue, Jersey City, N. J.

MoMahon, Prof. James. Cornell University, Ithaca, N. Y. 7 Central Avenue.

MoNeild, Prof. Malcolm. Lake Forest College, Lake Forest, Ill.

*Macaulay, Dr. F. S. The Chesters, Vicarage Road, East Sheen, London, S. W., England.

MacInnes, Asst. Prof. C..R. Princeton University, Princeton, N. J.

Mackinnon, Dr. Annie L. See Fitch, Mrs. Edward.

Maclaurin, Pres. R. C. Massachusetts Institute of Technology, Cambridge, Mass.

MacMillan, Asso. Prof. W. D. University of Chicago, Chicago, Ill. 5407 Woodlawn Avenue.

MaCNEISH, Dr. H. F. Instructor, College of the City of New York, New York, N. Y. 170 Vermilyea Avenue.

Maddison, Dr. Isabel. Recording Dean, Bryn Mawr College, Bryn Mawr, Pa.

Maddox, Asst. Prof. A. C. Oklahoma Agricultural and Mechanical College, Stillwater, Okla. 305 Ross Street.

MalloRy, V. S. East Orange High School, East Orange, N. J.

Maltbie, Dr. W. H. Attorney-at-Law. 726-728 Equitable Building, Baltimore, $\mathrm{Md}$.

Manning, Asso. Prof. H. P. Brown University, Providence, R. I.

Manning, Asso. Prof. W. A. Stanford University, Calif. 604 Tennyson Avenue, Palo Alto, Calif.

March, Asst. Prof. H. W. University of Wisconsin, Madison, Wis. 115 Summit Avenue.

MarkLex, Prof. J. L. University of Michigan, Ann Arbor, Mich. Geddes and Oxford Road.

Marshall, Prof. William. Purdue University, Lafayette, Ind. 1017 State Street.

Martin, Asso. Prof. Emilit N. Mount Holyoke College, South Hadley, Mass.

Martin, Prof. L. A. Stevens Institute of Technology, Hoboken, N. J. 911 Castle Point Terrace.

*Mason, Prof. MaX. University of Wisconsin, Madison, Wis.

Mason, Asso. Prof. T. E. Purdue University, Lafayette, Ind. 130 Andrew Place.

Matheson, Prof. John. Queen's University, Kingston, Ontario, Canada.

Mathews, R. M. Instructor, University of Minnesota, Minneapolis, Minn. 126 Folwell Hall.

Mathewson, Asst. Prof. L. C. Dartmouth College, Hanover, N. H. 
Meacham, Asst. Prof. E. D. University of Oklahoma, Norman, Okla. Faculty Exchange.

Melcher, GeORGe. Director, Bureau of Research and Efficiency, Public Schools, Kansas City, Mo. Room 22\%, Library Building.

MendizÁbat-Tamborres, Dr. Joaquin de. 5a calle de M. Doblado, No. 67, Mexico, D. F., Mexico.

Merrill, Asst. Prof. A. S. University of Montana, Missoula, Mont.

* Merrild, Prof. Helen A. Wellesley College, Wellesley, Mass.

Merriman, Dr. Mansfield. Consulting Engineer. 1071 Madison Avenue, New York, N. Y.

*Metzler, Prof. W. H. Syracuse University, Syracuse, N. Y. R. D. 1, Fayetteville, N. $Y$.

Miles, Asst. Prof. E. J. Sheffield Scientific School, Yale University, New Haven, Conn. 71 Marvel Road.

Miller, Dr. A. L. 25 Clinton Road, Brookline, Mass.

MILler, Prof. Bessie I. Rockford College, Rockford, Ill.

MILleR, E. A. 113 North Tioga Street, Ithaca, N. Y.

*Miller, Prof. G. A. University of Illinois, Urbana, Ill. 1203 West Illinois Street.

Miller, Prof. I. L. Carthage College, Carthage, Ill. 219 South Adams Street.

Miller, Prof. J. A. Swarthmore College, Swarthmore, Pa.

Milds, Vicente. Bureau of Insular Affairs, War Department, Washington, $\mathrm{D}$. C.

Milne, Prof. W. E. University of Oregon, Eugene, Ore.

Miser, Asst. Prof. W. L. Armour Institute of Technology, Chicago, III. Mitchell, Prof. B. E. Millsaps College, Jackson, Miss.

Mitchell, Prof. H. B. Columbia University, New York, N. Y. 80 Washington square.

Mrtcheld, Asst. Prof. H. H. University of Pennsylvania, Philadelphia, Pa. College Hall.

Mitchell, Asso. Prof. U. G. Kansas University, Lawrence, Kan. 1818 Massachusetts Street.

Moody, Prof. W. A. Bowdoin College, Brunswick, Me. 60 Federal Street.

Moore, Asso. Prof. C. L. E. Massachusetts Institute of Technology, Cambridge, Mass.

Moore, Asso. Prof. C. N. University of Cincinnati, Cincinnati, Ohio. 501 Sandheger Place.

*Moore, Prof. E. H. University of Chicago, Chicago, Ill. 5607 Kenwood Avenue.

Moore, Asst. Prof. R. L. University of Pennsylvania, Philadelphia, Pa. 5936 Washington Avenue.

Moors, Prof. E. E. Cornell College, Mount Vernon, Iowa. 319 North Fifth Avemue. 
Morehead, Asst. Prof. J. C. Carnegie Institute of Technology, Pittsburgh, Pa.

Moreno, Asso. Prof. H. C. Stanford University, Calif. P. O. Box 894.

Morgan, Asst. Prof. F. M. Dartmouth College, Hanover, N. H.

Moritz, Prof. R. E. University of Washington, Seattle, Wash. $4^{7} 05$ 21st Avenue, N.E.

MorLex, Prof. Frank. Johns Hopkins University, Baltimore, Md.

MORRIS, Dr. F. R. Instructor, University of California, Berkeley, Calif. 2034 Durant Avenue.

Morris, Prof. RICHARd. Rutgers College, New Brunswick, N. J. 76 Lincoln Avenue.

MORRISON, MONTFORD. Consulting Engineer, Victor Electric Corporation, 236 South Robey Street, Chicago, Ill.

Morrow, E. B. Gilman Country School, Roland Park, Md.

Monse, Dr. H. C. M. Instructor, Harvard University, Cambridge, Mass. 21 Conant Hall.

Moulton, Asso. Prof. E. J. Northwestern University, Evanston, Ill. 909 Colfax Street.

Moulton, Asso. Prof. F. R. University of Chicago, Chicago, Ill.

MuIR, Sir Thomas. Elmcote, Rondebosch, South Africa.

*MukнopâdHYÁx, Sir Asutosh. University of Calcutta, Calcutta, India. gry Russa Road, North, Bhowanipur.

Mullen, Dr. L. B. Girls' High School, Brooklyn, N. Y.

Mullins, Asst. Prof. G. W. Barnard College, Columbia University, New York, N. Y.

Murnaghan, Dr. F. D. Associate, Johns Hopkins University, Baltimore, Md.

MURRAY, Prof. D. A. MeGill University, Montreal, Canada.

Murrax, F. H. Harvard University, Cambridge, Mass. 33 Grays Hall.

Musselman, Dr. J. R. Instructor, Washington University, St. Louis, Mo.

Mrers, Prof. G. W. University of Chicago, Chicago, Ill. 1953 East M2d Street.

NeIkIRK, Asst. Prof. L. I. University of Washington, Seattle, Wash. 4723 21st Avenue, N. E.

NeLson, Dr. A. L. Instructor, University of Michigan, Ann Arbor, Mich. 1208 Olivia Avenue.

Nelson, Dr. C. A. Instructor, Kansas University, Lawrence, Kan. University Club.

Newcomer, Dr. H. S. 1112 Spaight Street, Madison, Wis.

NEWKIRK, Asst. Prof. B. L. University of Minnesota, Minneapolis, Minn.

Newson, Asst. Prof. Mary W. (Mrs. H. B.). Washburn College, Topeka, Kan. Whitin Hall.

NichоL, H. W. Research Engineer, Western Electric Company, 463 West Street, New York, N. Y. 
NoBle, Prof. C. A. University of California, Berkeley, Calif. 2224 Piedmont Avenue.

Northсотт, Asso. Prof. J. A. Syracuse University, Syracuse, N. Y. College of Applied Science.

Nowlan, Asst. Prof. F. S. Bowdoin College, Brunswick, Me. 15 Potter Street.

OdeLL, L. S. Manual Training High School, Brooklyn, N. Y.

OLDs, Prof. G. D. Amherst College, Amherst, Mass.

Osborne, Prof. G. A. Massachusetts Institute of Technology, Cambridge, Mass. 249 Berkeley Street, Boston, Mass.

Osgood, Prof. W. F. Harvard University, Cambridge, Mass. 74 Avon Hill Street.

O'Shaughnessy, Prof. Louis. Virginia Polytechnic Institute, Blacksburg, Va. P. O. Box 17\%.

Owens, Asst. Prof. F. W. Cornell University, Ithaca, N. Y. 110 Westburne Lane.

Owens, Dr. Helen B. (Mrs. F. W.). Instructor, Cornell University, Ithaca, N. Y. 110 Westbourne Lane.

Panswell, George. Civil Engineer. 212 West Fordham Road, New York, N. Y.

PAGE, Prof. J. M. University of Virginia, University, Va.

Palmer, Asso. Prof. C. I. Armour Institute of Technology, Chicago, Ill.

Palmer, E. S. Research Engineer, Winchester Repeating Arms Company, New Haven, Conn. 1623 Chapel Street.

Palmit, Prof. AnNa H. College for Women, Western Reserve University, Cleveland, Ohio, P. O. Box 371, Point Pleasant, $N$. J.

Parker, Prof. W. P. Pyeng Yang Union Christian College, Pyeng Yang, Korea.

Parsons, C. H. Massie School, Bronxville, N. Y.

*Partridge, Prof. E. A. West Philadelphia High School for Boys, 48th and Walnut Streets, Philadelphia, Pa.

Patten, Prof. W. E. Government Institute of Technology, Shanghai, China. Box \%0\%, American. Post Office.

Patterson, Dr. J. L. Chestnut Hill Academy, Chestnut Hill, Philadelphia, Pa.

Pattillio, Prof. N. A. Randolph-Macon Woman's College, Lynchburg, Va.

Peed, Prof. M. T. Emory University, Ga.

Pehrson, Asst. Prof. E. W. University of Utah, Salt Lake City, Utah. Peld, Dr. Alexander. Yarrow West, Bryn Mawr, Pa.

Pell, Asso. Prof. Anna J. (Mrs. Alexander). Bryn Mawr College, Bryn Mawr, Pa. Farrow West.

PFEIFFER, Dr. G. A. Instructor, Columbia University, New York, N. Y. 56 Anderson Avenue, Palisade, N. J. 
PhILliPs, Asst. Prof. H. B. Massachusetts Institute of Technology, Cambridge, Mass.

PIERCE, Dr. T. A. Instructor, University of Nebraska, Lincoln, Neb. 1029 South 15th Street.

Pierpont, Prof. James. Yale University, New Haven, Conn. 42 Mansfield Street.

Pitcher, Prof. A. D. Adelbert College, Western Reserve University, Cleveland, Ohio.

Plant, Prof. L. C. Michigan Agricultural College, East Lansing, Mich.

Plimpton, Dr. G. A. Ginn and Company, 70 Fifth Avenue, New York, N. Y.

Poor, Prof. C. L. C'olumbia University, New York, N. Y. 35 East 69 th Street.

PooR, Prof. J. M. Dartmouth College, Hanover, N. H.

Porter, Prof. M. B. University of Texas, Austin, Tex.

Posex, F. D. Continental Mexican Petroleum Company, Tampico, Tamps., Mexico. Apartado 652.

Pounder, I. R. Lecturer, University of Toronto, Toronto, Canada.

*Powers, R. E. Care of R. F. Watkins, Treasurer, Denver \& Rio Grande R. R. Co., Denver, Colo.

Prasad, Prof. Ganesh. Central Hindu College, Benares, India.

*Pupin, Prof. M. I. Columbia University, New York, N. Y.

Putnam, Prof. T. M. University of California, Berkeley, Calif.

Putnam, W. L. Attorney-at-Law. 60 State Street, Boston, Mass.

Ragsdale, Dr. Virginia. Associate, State Normal College, Greensboro, N. C. Jamestown, N. C.

Rambo, Asst. Prof. Susan M. Smith College, Northampton, Mass. 11 Barrett Place.

Ranum, Asst. Prof. Arthur. Cornell University, Ithaca, N. Y. 3 Central Avenue.

Rasor, Prof. S. E. Ohio State University, Columbus, Ohio. 1594 Neil Avenue.

Reaves, Prof. S. W. University of Oklahoma, Norman, Okla. 207 West. Boyd Street.

*Reddick, Prof. H. W. Cooper Union, New York, N. Y.

REED, Dr. F. W. Instructor, Cornell University, Ithaca, N. Y. 418 East Seneca Street.

ReEd, Asso. Prof. L. J. Johns Hopkins University, Baltimore, Md. 312 West Monument Street.

ReID, Prof. L. W. Haverford C'ollege, Haverford, Pa.

ReIlly, Asso. J. F. State University of Iowa, Iowa City, Iowa. 212 Physics Hall.

Reilly, Miss Marion. 2015 de Lancey Place, Philadelphia, Pa.

REMick, Prof. B. L. Kansas State Agricultural College, Manhattan, Kan. 613 Houston Street. 
Reynolds, Dr. C. N., Jr. Instructor, Wesleyan University, Middletown, Conn. 11 Pearl Street.

Reynolds, Asso. Prof. F. G. College of the City of New York, New York, N. Y.

RICE, C. M. 19 Pearl Street, Worcester, Mass.

RICE, L. H. Instructor, Massachusetts Institute of Technology, Cambridge, Mass. 143 Powder House Boulevard, West Somerville, Mass.

RICHARDSON, M. R. 618 McMannen Street, Durham, N. C.

*Richardson, Prof. R. G. D. Brown University, Providence, R. I.

*RICHMONd, H. W. Fellow and Lecturer, King's College, and University Lecturer, Cambridge University, Cambridge, England.

RIDER, Asst. Prof. P. R. Washington University, St. Louis, Mo.

RIETz, Prof. H. L. State University of Iowa, Iowa City, Iowa.

RIgas, Prof. N. C. Carnegie Institute of Technology, Pittsburgh, Pa.

RILey, Prof. J. L. Junior Agricultural and Mechanical College, Stephenville, Tex.

RIsley, Prof. W. J. James Millikin University; Decatur, Ill. 155 North Taylor Avenue.

RITT, Dr. J. F. Instructor, Columbia University, New York, N. Y. $15^{71}$ 51st Street, Brooklyn, N. Y.

RobBINs, Dr. R. B. Instructor, University of Michigan, Ann Arbor, Mich. 1113 Ferdon Road.

Roberts, Prof. Maria M. Iowa State College, Ames, Iowa. 219 Ash Avenue.

Robinson, L. B. 306 East 22d Street, Baltimore, Md.

*Rok, Prof. E. D., Jr. Syracuse University, Syracuse, N. Y. 123 West Ostrander Avenue.

*Roe, Dr. Josephine R. (Mrs. E. D.). 123 West Ostrander Avenue, Syracuse, N. Y.

Roever, Prof. W. H. Washington University, St. Louis, Mo.

Roosevelt, G. E. 30 Pine Street, New York, N. Y.

Rоoт, Prof. R. E. U. S. Naval Academy, Annapolis, Md. 7 Franklin Street.

Rorer, Dr. J. T. William Penn High School, Philadelphia, Pa. 333 North 34th Street.

Rosenbach, J. B. University of Illinois, Urbana, ml. 606 South Goodwin Street.

RosenbaUm, Dr. JosePh. Instructor, Rosenbaum School, Milford, Conn.

Rothrock, Prof. D. A. Indiana University, Bloomington, Ind. 1000 Atwater Avenue.

RothsCHILD, S. Z. Actuary, Sun Life Insurance Company, Sun Life Building, Baltimore, Md.

Rowe, Asso. Prof. J. E. Pennsylvania State College, State College, Pa. 142 South Frazier Street.

Running, Asso. Prof. T. R. University of Michigan, Ann Arbor, Mich. 1019 Michigan Avenue. 
Rusk, Prof. W. J. Grinnell C'ollege, Grinnell, Iowa. 102\% Park Street. Russeli, Asso. Prof. W. P. Pomona College, Claremont, Calif.

Rutledge, Dr. George. Instructor, Massachusetts Institute of Technology, Cambridge, Mass. 28 Chatham Street.

SAFrord, Asst. Prof. F. H. University of Pennsylvania, Philadelphia, $\mathrm{Pa}$.

SAUREL, Prof. P. L. College of the City of New York, New York, N. Y. SCARBoRough, J. B. Instructor, U. S. Naval Academy, Annapolis, Md. P. O. Box 332.

SCARBorough, Prof. J. H. Missouri State Normal School, Warrensburg, Mo.

*SCHMIEDEI, OSCAR. Parsons College, Fairfield, Iowa. 100\% North Fourth Street.

SchUYler, Elmer. Bay Ridge High School, 67th Street and Fourth Avenue, Brooklyn, N. Y.

Schwatt, Prof. I. J. University of Pennsylvania, Philadelphia, Pa. 1226 South 58th Street.

Schweitzer, Dr. A. R. 452 Oakdale Avenue, Chicago, Ill.

Scott, Prof. Charlotre A. Bryn Mawr College, Bryn Mawr, Pa. 233 Roberts Road.

Seetr, Dr. Caroline E. 501 West 116th Street, New York, N. Y.

Stauier, Rev. J. A. M. J. DE. 114 Rue du Bac, Paris, 7e, France.

Sellew, Prof. G. T. Knox College, Galesburg, Ill.

Sharpe, Prof. F. R. Cornell University, Ithaca, N. Y. 213 Mitchell Street.

SHAw, Prof. J. B. University of Illinois, Urbana, Ill. 901 West California Avenue.

SHEFFer, Dr. H. M. Lecturer, Harvard University, Cambridge, Mass. Emerson Hall.

Shet.don, Prof. E. W. University of Alberta, Edmonton South, Alberta, Canada.

SHoRT, R. L. Director of High Schools. Lakewood, Ohio.

Shugers, Asst. Prof. S. P. University of Pennsylvania, Philadelphia, Pa. Box 13, College Hall.

Shumway, Asso. Prof. R. R. University of Minnesota, Minneapolis, Minn. 3844 Thomas Avenue, South.

Siceloff, Asst. Prof. L. P. Columbia University, New York, N. Y.

Silverman, Asst. Prof. L. L. Dartmouth College, Hanover, N. H.

Srmon, Dr. W. G. Instructor, Adelbert College, Western Reserve University, Cleveland, Ohio. 1581 Crawford Road.

Simonds, Dr. E. F. "Adair," Lamrock Avenue, Bondi, New South Wales, Australia.

Simpson, Asst. Prof. C. G. Pennsylvania State College, State College, Pa. 306 South Burrows Street. 
Simpson, Prof. T. M. University of Florida, Gainesville, Fla. 311 West Church Street.

Sinclair, Asso. Prof. Mary E. Oberlin College, Oberlin, Ohio. 69 North Cedar Avenue.

${ }^{*}$ Sisam, Prof. C. H. Colorado College, Colorado Springs, Colo. 1536 Wood Avenue.

Skiles, Asso. Prof. W. V. Georgia School of Technology, Atlanta, Ga. 306 Myrtle Street.

Skinner, Asso. Prof. E. B. University of Wisconsin, Madison, Wis. 210 Lathrop Street.

Slaught, Prof. H. E. University of Chicago, Chicago, Ill. $5548 \mathrm{Ken}$ wood Avenue.

Slepian, Dr. JosePH. 433 Ross Avenue, Wilkinsburg, Pa.

${ }^{*}$ Slichter, Prof. C. S. University of Wisconsin, Madison, Wis. 636 Frances Street.

Slocum, Prof. S. E. University of Cincinnati, Cincinnati, Ohio.

SmaIL, Dr. L. L. Instructor, University of Washington, Seattle, Wash. 2121 East 55th Street.

Smith, Asso. Prof. A. W. Colgate University, Hamilton, N. Y.

${ }^{*}$ Smith, Asso. Prof. Crara E. Wellesley College, Wellesley, Mass. Shafer Hall.

*Smith, Prof. D. E. Teachers College, Columbia University, New York, N. Y.

Sмith, Asst. Prof. D. M. Georgia School of Technology, Atlanta, Ga.

Smith, Asso. Prof. Edwin R. Pennsylvania State College, State College, Pa. Room 105, Main.

Smith, Eugene R. Headmaster, The Park School, Baltimore, Md.

Smith, Miss Gertrude. Instructor, Vassar C'ollege, Poughkeepsie, N. Y.

SмIтн, Dr. G. W. Instructor, University of Kentucky, Lexington, Ky. 336 Harrison Avenue.

Smith, Asst. Prof. I. W. North Dakota Agricultural College, Fargo, N. Dak. 1126 13th Street, North.

SмirH, Prof. P. F. Sheffield Scientific School, Yale University, New Haven, Conn. 330 Willow Street.

Smith, Prof. SARah E. Mount Holyoke College, South Hadley, Mass.

Smith, Prof. W. M. Lafayette College, Easton, Pa.

Snelling, Prof. C. M. University of Georgia, Athens, Ga. P. O. Box 283.

Snook, T. E. Architect and Engineer. 261 Broadway, New York, N. Y.

SNyder, Prof. M. B. Central High School, Philadelphia, Pa. 240\% North Broad Street.

SNYder, Prof. VIraIL. Cornell University, Ithaca, N. Y. 214 University Avenue.

Sommerville, Prof. D. M. Y. Victoria University College, Wellington, New Zealand. 
Sparrow, Asso. Prof. C. M. University of Virginia, University, Va.

SPERRY, Dr. PaUline. Instructor, University of California, Berkeley, Calif. 1610 Leroy Avenue.

SPItZer, George. Dairy Chemist, Purdue University, Lafayette, Ind. 1000 Seventh Street, West Lafayette.

Stager, Prof. H. W. Fresno Junior College, Fresno, Calif. 265 Howard Street.

*Stecker, Prof. H. F. Pennsylvania State College, State College, Pa. 306 Miles Street.

Steimlex, L. L. Instructor, University of Illinois, Urbana, Ill. 1008 West Green Street.

Steinmetz, Prof. C. P. Union College, Schenectady, N. Y. Chief Consulting Engineer, General Electric Company. Wendell Avenue.

Stephens, Prof. R. P. University of Georgia, Athens, Ga.

Stetson, Dr. J. M. Instructor, Sheffield Scientific School, Yale University, New Haven, Conn. 885 Orange Street.

Stone, Prof. ORmond. University of Virginia, University, Va. Clifton Station, $\nabla a$.

Stone, Asst. Prof. R. B. Purdue University, Lafayette, Ind. 307 Russell Street, West Lafayette.

Story, Prof. W. E. Clark University, Worcester, Mass. 17 Hammond Street.

Stouffer, Asso. Prof. E. B. Kansas University, Lawrence, Kan. 947 Louisiana Street.

Stoughton, Miss Leila R. 270 Everit Street, New Haven, Conn.

Stromquist, Prof. C. E. University of Wyoming, Laramie, Wyo.

Strong, Dr. W. M. Associate Actuary, Mutual Life Insurance C'ompany, 32 Nassau Street, New York, N. Y.

Sullivan, Asst. Prof. C. T. MoGill University, Montreal, Canada. Engineering Building.

Swartzel, Prof. K. D. Ohio State University, Columbus, Ohio. 1952 Iuka Avenue.

*SwIFT, Prof. EliJah. University of Vermont, Burlington, Vt. 415 South Willard Street.

*Taber, Prof. Henry. Clark University, Worcester, Mass.

*TANNER, Prof. J. H. Cornell University, Ithaca, N. Y. Cayuga Heights.

Tanzola, J. J. Instructor, Cooper Union, New York, N. Y. 162 Ninth Street, Brooklyn, N. $Y$.

Tappan, Asst. Prof. A. Helen. Iowa State College, Ames, Iowa. 2402 Lincoln Way.

TAYLoR, Dr. E. H. Instructor, Eastern State Normal School, Charleston, 111.

TAYıOR, Prof. J. M. Colgate University, Hamilton, N. Y.

TAYLOR,'Dr. J. S. Instructor, Massachusetts Institute of Technology, Cambridge, Mass. 
TerRILL, H. M. Columbia University, New York, N. Y. 160 Seminary Avenue, Rahway, N.J.

Thomas, Prof. Evan. College of Engineering, University of Vermont, Burlington, Vt. 187 Loomis Street.

*Thompson, Prof. H. D. Princeton University, Princeton, N. J. 11 Morven Street.

Thompson, J. S. Assistant Actuary, Mutual Life Insurance Company, 34 Nassau Street, New York, N. Y.

Touton, Dr. F. C. Supervisor of High Schools, State Department of Public Instruction, Madison, Wis.

Townsend, Prof. E. J. University of Illinois, Champaign, Ill. 510 John Street.

Tracey, Asst. Prof. J. I. Yale University, New Haven, Conn. 314 Norton Street.

TrIP., Asso. Prof. M. O. University of Maine, Orono, Me.

TrLer, Prof. H. W. Massachusetts Institute of Technology, Cambridge, Mass.

UNDERHILl, Asst. Prof. A. L. University of Minnesota, Minneapolis, Minn. 911 Fifth Street, S.E.

Upton, Asso. Prof. C. B. Teachers College, Columbia University, New York, N. Y.

Urner, Dr. S. E. P. O. Box 451, Route A, Mountain View, Calif.

*Van Benschoten, Prof. Anna L. Wells College, Aurora, N. Y.

VAN DER VRIES, Prof. J. N. Chamber of Commerce of the United States, 801 Otis Building, Chicago, Ill.

VANDIVER, H. S. Instructor, Cornell University, Ithaca, N. Y. 128 Eddy Street.

*Van Orstrand, C. E. Physical Geologist, U. S. Geological Survey, and Lecturer, George Washington University, Washington, D. C. 1607 31st Street, N. W.

VAN Vleck, Prof. E. B. University of Wisconsin, Madison, Wis. 519 North Pinckney Street.

*Veblen, Prof. Oswald. Princeton University, Princeton, N. J.

VedDER, Asst. Prof. J. N. Union College, Schenectady, N. Y.

Vivian, Prof. Roxana H. Wellesley C'ollege, Wellesley, Mass.

Voltterra, Prof. Vito. University of Rome, Rome, Italy. Via in Lucina, $1 \%$.

Waddell, Miss Mary E. G. 655 Spadina Avenue, Toronto, Canada.

WaHLin, Asst. Prof. G. E. University of Illinois, Urbana, Ill. $60^{7}$ West Nevada Street.

WALSH, J. L. Harvard University, Cambridge, Mass. B 12, Gore Hall. 
Washburne, A. C. Actuary, Berkshire Life Insurance Company, Pittsfield, Mass.

WatKeys, Prof. C. W. University of Rochester, Rochester, N. Y.

Watson, Prof. G. N. University of Birmingham, Edmund Street, Birmingham, England.

Wear, Asso. Prof. L. E. Throop College, Pasadena, Calif. 261 South Bonnie Avenue.

Weaver, Dr. J. H. Instructor, Ohio State University, Columbus, Ohio. Hilliard, Ohio.

WebB, H. E. Central High School, Newark, N. J. 12 Irving Place, Summit, $N . J$.

Webster, Prof. A. G. Clark University, Worcester, Mass. 66 West Street.

WeChSLER, A. L. 895 West End Avenue, New York, N. Y.

Weiderburn, Asst. Prof. J. H. M. Princeton University, Princeton, N. J. P. O. Box 53 .

Weeks, Dr. Eula A. Grover Cleveland High School, St. Louis, Mo.

WeEks, R. W. Chief Actuary, New York Life Insurance Company, 346 Broadway, New York, N. Y.

WEIDA, F. M. Instructor, State University of Iowa, Iowa City, Iowa. P. O. Box 371.

WeLD, Prof. L. G. Pullman Free School of Manual Training, Pullman, III.

Wells, Asst. Prof. Mary E. Vassar College, Poughkeepsie, N. Y.

Wentworth, George. 1688 Beacon Street, Brookline, Mass.

Wester, Asst. Prof. C. W. State Teachers College, Cedar Falls, Iowa.

*Western, Dr. A. E. 35 Essex Street, Strand, London, W. C. 2, England.

Westrall, Asso. Prof. W. D. A. University of Missouri, C'olumbia, Mo. 915 University Avemue.

Westlund, Prof. JAcoв. Purdue University, Lafayette, Ind. 5436 University Avenue, Chicago, Ill.

Wetzes, R. A. Instructor, College of the City of New York, New York, N. Y. 218 Tecumseh Avenue, Mount Vernon, N. Y.

Whiте, Prof. H. S. Vassar College, Poughkeepsie, N. Y. Box 41, Vassar College.

White, Asst. Prof. Marion B. Carleton College, Northfield, Minn. 408 East Second Street.

Whitrord, Asst. Prof. E. E. College of the City of New York, New York, N. Y. 3681 Broadway.

Whitney, A. W. General Manager, National Workmen's Compensation Service Bureau, 15 Park Row, New York, N. Y.

*Whittaker, Prof. E. T. University of Edinburgh, Edinburgh, Scotland. 35 George Square.

*WhitTemore, J. K. Instructor, Sheffeld Scientific School, Yale University, New Haven, Conn. 
WIENER, Dr. NoRBert. Instructor, Massachusetts Institute of Technology, Cambridge, Mass. 29 Sparkes Street.

Wilczynski, Prof. E. J. University of Chicago, Chicago, Ill.

WILdER, Asst. Prof. C. E. Northwestern University, Evanston, Ill. 749 Sherman Avenue.

WILEx, Prof. F. B. Denison University, Granville, Ohio.

Willard, Prof. J. M. Pennsylvania State College, State College, Pa.

Williams, Dr. A. R. Instructor, U. S. Shipping Board Navigation School, Portland, Ore. T. M. C. A. Building.

Williams, Prof. C. B. Kalamazoo College, Kalamazoo, Mich. 214 Stuart Avenue.

Williams, Miss Ella C. Miss Spence's School, 30 West 55th Street, New York, N. Y.

Williams, Dr. Emiry C. (Mrs. W. H.). 24 West 58th Street, New York, N. Y.

Williams, Prof. F. B. Clark University, Worcester, Mass.

Williams, Prof. J. E. Virginia Polytechnic Institute, Blacksburg, Va.

Williams, Asst. Prof. K. P. Indiana University, Bloomington, Ind. 405 North Indiana Avenue.

Williams, Prof. W. H. State Normal School, Platteville, Wis.

Willson, Prof. F. N. Princeton University, Princeton, N. J. P. 0. Box 63.

Wilson, Asso. Prof. A. H. Haverford College, Haverford, Pa.

*Wilson, Asso. Prof. D. T. C'ase School of Applied Science, Cleveland, Ohio.

WiLson, Prof. E. B. Massachusetts Institute of Technology, Cambridge, Mass.

WILson, Dr. L. T. Instructor, U. S. Naval Academy, Annapolis, Md. 312 West Street.

Wilson, Prof. N. R. University of Manitoba, Winnipeg, Manitoba, Canada.

Wilson, Asst. Prof. R. E. Northwestern University, Evanston, Ill.

WILson, Asst. Prof. W. A. Yale University, New Haven, Conn. 228 Parak Street.

Winger, Asst. Prof. R. M. University of Washington, Seattle, Wash.

Wolfe, Dr. C. L. E. Dean, Junior College, Santa Rosa, Calif. 1063 Fourth Street.

Wolfr, Prof. H. C. Drexel Institute, Philadelphia, Pa.

Wood, Prof. Ruth G. Smith College, Northampton, Mass. 249 Crescent Street.

Woops, Prof. B. M. University of California, Berkeley, Calif.

Woods, Prof. F. S. Massachusetts Institute of Technology, Cambridge, Mass.

*Woodward, Pres. R. S. Carnegie Institution, Washington, D. C.

Worthington, Dr. Euphemia R. Instructor, Wellesley College, Wellesley, Mass. 10 Waban Street. 
WRIGHT, Prof. W. L. Lincoln University, Pa.

YANNEY, Prof. B. F. College of Wooster, Wooster, Ohio.

Yeaton, Prof. C. H. School of Engineering of Milwaukee, Milwaukee, Wis. 373 Broadway.

Yen, Prof. ChIA-ChEOW. Tangshan Engineering College, Tangshan, North China.

Young, Prof. A. E. Miami University, Oxford, Ohio.

Younc, Prof. J. W. Dartmouth College, Hanover, N. H.

Young, Asso. Prof. J. W. A. University of Chicago, Chicago, Ill. 542\% Blackstone Avenue.

YowelL, Dr. E. I. First Astronomer, Cincinnati Observatory, Cincinnati, Ohio. Griest and Corbett Avenues.

Zehring, Asst. Prof. W. A. Purdue University, Lafayette, Ind. 303 Russell Street.

Zervos, Prof. Panatotis. University of Athens, Athens, Greece. Rue Sozopoleos 88.

Zrwet, Prof. Alexander. University of Michigan, Ann Arbor, Mich. 644 Tappan Avenue.

Number of members, January 1, 1918................. 739

Members admitted during the year $1918 \ldots \ldots \ldots \ldots \ldots \ldots \ldots \ldots . \ldots \ldots$

Members withdrawing during the year $1918 \ldots \ldots \ldots \ldots \ldots \ldots \ldots, 40$

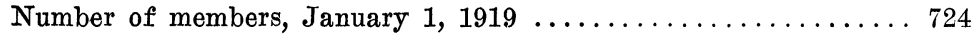

Members admitted during the year $1919 \ldots \ldots \ldots \ldots \ldots \ldots \ldots \ldots . \ldots \ldots$

Members withdrawing during the year $1919 \ldots \ldots \ldots \ldots \ldots \ldots, 16$

Number of members, January 1, $1920 \ldots \ldots \ldots \ldots \ldots \ldots \ldots \ldots \ldots \ldots$

Number of life members, January $1,1920 \ldots \ldots \ldots \ldots \ldots \ldots \ldots .60$ 